\title{
Defining Polysaccharide Antibody Deficiency: Measurement of Anti-Pneumococcal Antibodies and Anti-Salmonella typhi Antibodies in a Cohort of Patients with Recurrent Infections
}

\author{
Giorgia Bucciol ${ }^{1,2} \cdot$ Heidi Schaballie ${ }^{1,2,3} \cdot$ Rik Schrijvers $^{4} \cdot$ Barbara Bosch $^{2} \cdot$ Marijke Proesmans $^{2} \cdot$ Kris De Boeck $^{2}$. \\ Mieke Boon ${ }^{2} \cdot$ François Vermeulen $^{2} \cdot$ Natalie Lorent $^{5} \cdot$ Doreen Dillaerts $^{6} \cdot$ Bjørn Kants $\varnothing^{7}$. \\ Charlotte Svaerke Jørgensen ${ }^{7} \cdot$ Marie-Paule Emonds $^{8} \cdot$ Xavier Bossuyt $^{6,9} \cdot$ Leen Moens $^{1} \cdot$ Isabelle Meyts ${ }^{1,2}$ (i)
}

Received: 2 August 2019 / Accepted: 11 September 2019

(C) Springer Science+Business Media, LLC, part of Springer Nature 2019

\begin{abstract}
Background The correlation between different methods for the detection of pneumococcal polysaccharide vaccine (PPV) responses to diagnose specific polysaccharide antibody deficiency (SAD) is poor and the criteria for defining a normal response lack consensus. We previously proposed fifth percentile (p5) values of PPV responses as a new cutoff for SAD.

Objective To analyze the association of SAD (determined by either World Health Organization (WHO)-standardized ELISA or multiplex bead-based assay) with abnormal response to Salmonella (S.) typhi Vi vaccination in a cohort of patients with recurrent infections.

Methods Ninety-four patients with a clinical history suggestive of antibody deficiency received PPV and S. typhi Vi vaccines. Polysaccharide responses to either 3 or 18 pneumococcal serotypes were measured by either the WHO ELISA or a multiplex inhouse bead-based assay. Anti-S. typhi Vi IgG were measured by a commercial ELISA kit. Allohemagglutinins (AHA) were measured by agglutination method.

Results Based on the American Academy of Allergy, Asthma and Immunology (AAAAI) criteria for WHO ELISA, 18/94 patients were diagnosed with SAD and 22/93 based on serotype-specific p5 cutoffs for bead-based assay. The association between the two methods was significant, with 10 subjects showing abnormal response according to both techniques. Abnormal response to S. typhi Vi vaccination was found in 7 patients, 6 of which had SAD. No correlation was found between polysaccharide response and AHA, age, or clinical phenotype.
\end{abstract}

Giorgia Bucciol and Heidi Schaballie equally contributed to this work.

Electronic supplementary material The online version of this article (https://doi.org/10.1007/s10875-019-00691-8) contains supplementary material, which is available to authorized users.

Isabelle Meyts

isabelle.meyts@uzleuven.be

1 Inborn errors of immunity, Department of Microbiology, Immunology and Transplantation, KU Leuven, Leuven, Belgium

2 Department of Pediatrics, University Hospitals Leuven, Herestraat 49, 3000 Leuven, Belgium

3 Present address: Department of Pediatric Pulmonology, Infectious Diseases and Primary Immunodeficiencies, Ghent University Hospital, Ghent, Belgium

4 Department of Microbiology, Immunology and Transplantation, Research group Allergy and Clinical Immunology, KU Leuven, Leuven, Belgium
5 Department of Respiratory Diseases, University Hospitals Leuven, Leuven, Belgium

6 Clinical and Diagnostic Immunology, Department of Microbiology, Immunology and Transplantation, KU Leuven, Leuven, Belgium

7 Virus and Microbiological Special Diagnostics, Statens Serum Institut, Copenhagen, Denmark

8 Histocompatibility and Immunogenetic Laboratory, Red Cross Flanders, Mechelen, Belgium

9 Department of Laboratory Medicine, University Hospitals Leuven, Leuven, Belgium 
Conclusion The lack of evidence-based gold standards for the diagnosis of SAD represents a challenge in clinical practice. In our cohort, we confirmed the insufficient correlation between different methods of specific PPV response measurement, and showed that the $S$. typhi Vi response was not contributive. Caution in the interpretation of results is warranted until more reliable diagnostic methods can be validated.

Keywords Pneumococcal polysaccharide vaccine $\cdot$ specific antibody deficiency $\cdot$ polysaccharide antibody deficiency $\cdot$ primary immunodeficiency $\cdot$ antibody deficiency $\cdot$ Salmonella typhi $\cdot$ SAD $\cdot$ allohemagglutinins

\section{Introduction}

Specific polysaccharide antibody deficiency (SAD) is a phenotypic entity characterized by deficient antibody production directed against the capsular polysaccharides of encapsulated bacteria, such as Streptococcus pneumoniae, Staphylococcus aureus, and Haemophilus influenzae [1-3]. The production of these antibodies is essential for optimal host defense. The typical signs and symptoms of SAD are recurrent respiratory tract infections (mostly otitis media, sinusitis, and pneumonia) in the presence of normal levels of immunoglobulin (Ig)G, $\operatorname{IgA}, \operatorname{IgM}$, and IgG subclasses, while more severe infections, such as meningitis, septic arthritis, abscesses, and sepsis, are rare [1-4]. SAD can be isolated in patients with an otherwise intact immune system or make part of more severe inborn errors of immunity. For example, SAD is a manifestation of common variable immunodeficiency (CVID), several combined immunodeficiencies (e.g., 22q11 deletion, WiskottAldrich syndrome, ataxia-telangiectasia), defects of nuclear factor (NF)-KB signaling pathway (e.g., IKBKG, NFKB1, $N F K B 2$ ), cystic fibrosis, asplenia, and Down syndrome $[2,4]$.

The diagnosis of SAD is classically based on the measurement of serotype-specific IgG against pneumococcal polysaccharide vaccines (PPV) by an enzyme-linked immunosorbent assay (ELISA), standardized by the World Health Organization (WHO) in 1995 [5]. The American Academy of Allergy, Asthma and Immunology (AAAAI) defines a normal response to PPV as a post-vaccination IgG concentration $\geq 1.3 \mu \mathrm{g} / \mathrm{ml}$ and a two-fold increase of the post-vaccination concentration when compared with the pre-vaccination concentration (unless pre-vaccination $\mathrm{IgG}>4 \mu \mathrm{g} / \mathrm{ml}$ ) [6]. A healthy subject should achieve these values for at least $70 \%$ of tested serotypes (50\% for children aged 2-5 years). Multiplex bead-based assays for specific PPV IgG determination are increasingly being utilized, thanks to their ease of use, fast turn-around time, and low cost compared with the WHO ELISA. However, the poor correlation of results from multiplex and ELISA assays, low reproducibility between different laboratories, and lack of standardized thresholds to define a normal or abnormal response remain problematic [1, 2, 7-9]. Other tests have been proposed as complementary or as alternative to pneumococcal polysaccharide response for the diagnosis of SAD, such as measurement of allohemagglutinins
(AHA) and of anti-Salmonella (S.) typhi Vi antibodies, which are both anti-polysaccharide antibodies [6, 10-14].

We previously established cutoff values for antipneumococcal polysaccharide antibodies measured by WHO ELISA and by an in-house Luminex bead-based assay, for anti-S. typhi $\mathrm{Vi}$ antibodies and for AHA in a large cohort of healthy volunteers [14]. We demonstrated that the AAAAI criteria resulted in a $30 \%$ of healthy subjects being defined as $\mathrm{SAD}$, while applying population-based fifth percentile (p5) values resulted in only $2-4 \%$ SAD, using the WHO ELISA or bead-based assay respectively [14]. In this study, we applied these thresholds to patients with symptoms compatible with antibody defects to determine the proportion of subjects with SAD as identified by the various methods, and to study the potential added value of measuring $S$. typhi responses. Moreover, we tested the correlation between the different assays/criteria used.

\section{Materials and Methods}

\section{Study Design}

We conducted a prospective cohort study in patients referred to the Pediatric or Adult Immunology Clinic of University Hospitals Leuven, Belgium. The study was approved by the ethical committee of the hospital and written informed consent was obtained from the patients or their parents. Patients were enrolled when they met the following inclusion criteria: (i) medical history suggestive of antibody deficiency (including recurrent lower and upper respiratory tract infections, otitis with or without otorrhea, skin/soft tissues/invasive bacterial infections), so that assessment of polysaccharide antibody response was indicated for the clinical care of the patient; (ii) age between 2 and 55 years. Exclusion criteria were as follows: (i) vaccination with an unconjugated polysaccharide Salmonella vaccine or pneumococcal vaccine in the previous 5 years; (ii) previous allergic reaction to any vaccine; (iii) pregnancy at the time of enrolment. A blood sample was obtained at inclusion to determine baseline antibody levels against PPV, S. typhi Vi, AHA, and blood group. Typhim Vi ${ }^{\mathrm{TM}}$ (Sanofi Pasteur, Lyon, France) and Pneumovax 23 ${ }^{\mathrm{TM}}$ (Merck Sharp en Dohme B.V., 
Haarlem, the Netherlands) vaccines were administered by intramuscular injection at the same visit in two distinct sites (right and left deltoid muscle). Information on the clinical history and potential previous contact with S. typhi was obtained by a physician via a standardized case record form. Four weeks after vaccination, a second blood sample was obtained for post-vaccination antibody concentrations. Preand post-vaccination blood was separated by centrifugation and serum was stored at $-20{ }^{\circ} \mathrm{C}$ until simultaneous analysis of specific IgG. Median time in storage was 6 months, with a range of 1-12 months.

\section{Antibody Response to PPV}

Anti-pneumococcal polysaccharide IgG against three serotypes $(8,9 \mathrm{~N}$, and $15 \mathrm{~B})$ that are not included in the existing pneumococcal conjugated vaccines (PCV) were measured by the third-generation WHO ELISA, incorporating adsorption of samples with cell wall polysaccharide and capsular polysaccharide 22F [13]. Only three serotypes were included based on standard laboratory practice in our hospital. We previously demonstrated high precision of the ELISA assay in our laboratory $[15,16]$. Antibody measurement of specific anti-pneumococcal IgG antibodies to serotypes 1, 3, 4, 5, $6 \mathrm{~B}, 7 \mathrm{~F}, 9 \mathrm{~V}, 14,18 \mathrm{C}, 19 \mathrm{~A}, 19 \mathrm{~F}$, and 23F (included in both 13-valent PCV and PPV), and of serotypes 8, 9N, 10A, 17F, 20 , and $33 \mathrm{~F}$ (included in PPV but not in any PCV) was performed at the Statens Serum Institute using an in-house Luminex bead-based assay, based on the procedure described by Lal et al. [17]. This method permits the simultaneous measurement of all 18 serotype-specific IgG in a single well. Briefly, pneumococcal polysaccharides purchased from LGC Standards (American Type Culture Collection, VA, USA) or from SSI Diagnostica (Hilleroed, Denmark) were conjugated to poly-1-lysine and then covalently bound to carboxylated microspheres (Luminex, TX, USA). Serum samples (and the 89SF standard) were pre-adsorbed in adsorbance buffer containing CWPS Multi (SSI Diagnostica, Hilleroed, Denmark) and then incubated with the conjugated microspheres, followed by incubation with R-phycoerythrin-conjugated antihuman IgG (Jackson ImmunoResearch laboratories, West Grove, PA, USA). Finally, the microspheres were read on a Bio-Plex 200 system (Bio-Rad, Hercules, CA, USA). Data were acquired using Bio-Plex Manager 5.0 (Bio-Rad Hercules, CA, USA). Pneumococcal polysaccharide serum calibrated to the FDA 89SF reference serum was used as a reference [18]. Serum IgG concentrations were calculated using a standard curve of median fluorescent intensity (MFI) against expected IgG concentration for FDA 89SF and converted to micrograms per milliliter. Pre- and postimmunization samples were analyzed on the same plate. Each sample was analyzed in duplicate, and analysis was repeated if the coefficient of variation between duplicates was above $10 \%$. To analyze the results for the serotypes measured by Luminex bead-based assay, patients were divided into three groups according to the year of birth, in order to control for previous vaccination with $\mathrm{PCV}$, offered to all children in Belgium since 2004 (PCV7) or 2011 (PCV13). For patients born before 2004, all serotypes were included in the analysis, for those born between 2004 and 2011, only the 11 serotypes not present in PCV7, and for those born after 2011, only the 6 not present in PCV13.

\section{Antibody Response to S. typhi Vi Vaccine}

Specific antibodies to $S$. typhi Vi vaccine were measured using a commercially available ELISA kit (VaccZyme ${ }^{\mathrm{TM}}$ antiS. typhi Vi human IgG EIA kit from The Binding Site Group Ltd., Birmingham, UK). Samples, standards, and quality controls were run in duplicate following the manufacturer's instructions. Pre- and post-vaccination samples were analyzed on a single plate in a single run. The results were expressed as units per milliliter (range 7.4-600 U/ml). Samples resulting in a value below the lower limit of detection $(<7.4 \mathrm{U} / \mathrm{ml})$ were set to $3.7 \mathrm{U} / \mathrm{ml}$. Values above the upper limit of detection $(600 \mathrm{U} / \mathrm{ml})$ are reported as $600 \mathrm{U} / \mathrm{ml}$.

\section{Detection of Allohemagglutinins}

Blood group and AHA were determined on pre-vaccination EDTA blood samples. Anti-A and anti-B IgG and IgM (AHA) were determined by the Immunohematology Laboratory of the Red Cross Flanders, using column agglutination technology (Bio-Rad®) with LISS Coombs columns and neutral columns, respectively, as previously described [13].

\section{Statistical Analysis}

Correlation between continuous variables (IgG values, number of infections) was analyzed by linear regression. Fisher's exact test of independence was used to calculate the statistical significance of the correlation between categorical variables (S. typhi antibody response, SAD diagnosis, AHA response, age groups). Statistical analyses were performed with GraphPad Prism (v. 8.0.0, CA, USA).

\section{Results}

\section{Study Population}

Ninety-nine patients were included. Five did not conclude the study: three because they did not receive the Typhim $\mathrm{Vi}^{\mathrm{TM}}$ vaccine and two because they withdrew voluntarily. Analyses were thus performed in 94 patients. 
The cohort consisted of 79 children between 2 and 18 years old and 15 adults between 20 and 52 years old. The median age was 4 years (range 2-52 years, interquartile range 2-9.5 years) and the male to female ratio was 1:0.8 (52 males and 42 females). Most subjects were of European descent, except 3 of NorthAfrican, 1 of Persian, and 1 of Turkish descent. The median interval between the vaccination and the post-vaccination blood sample was 28 days (interquartile range 27-31 days).

Most patients suffered from upper and/or lower respiratory tract infections. Thirty-seven patients (39\%) manifested one or more episodes of pneumonia and one had computed tomography-confirmed bronchiectasis; recurrent upper respiratory tract infections were reported in 91 patients $(97 \%), 77$ of whom $(82 \%)$ suffered from more than four episodes per year. Recurrent otitis was present in 44 patients (47\%), 34 of whom $(36 \%)$ had more than 4 episodes in the last 5 years. Eighteen patients (19\%) suffered from invasive infections, including sepsis, meningitis, septic arthritis, deep abscesses, or pyelonephritis; one patient had recurrent severe herpes simplex and varicella-zoster cutaneous infections; two had chronic mucocutaneous candidiasis (CMC); and four patients suffered from recurrent superficial bacterial infections (abscesses/cellulitis).

Other manifestations included the following (each only present in a single different subject): chronic fatigue and fibromyalgia, granulomatous hepatitis, severe eczema and allergy, Saethre-Chotzen syndrome (craniosynostosis), and recurrent parotitis.

Further analyses as part of the clinical and diagnostic workup of the subjects and not directly related to this study identified 4 patients with an underlying condition affecting the immune system: one with cystic fibrosis, one with trisomy 21 , one with complement factor 8 deficiency, and one with inhibitor of nuclear factor kappa B kinase subunit beta (IKK2 or IKK- $\beta$ ) deficiency.

No serious or severe vaccine-related adverse events were reported. Minor adverse events included fever $(n=2)$, local tenderness and redness at the site of PPV injection $(n=5)$, diffuse skin rash $(n=2)$, and syncope at the time of injection $(n=2)$.

\section{Pneumococcal Polysaccharide Antibody Response}

PPV antibody response was determined for all 94 subjects by WHO ELISA and for 93 subjects by bead-based assay.

Based on the AAAAI guidelines for SAD on WHO ELISA, 4 subjects responded to $0 / 3$ and 14 to $1 / 3$ tested serotypes $(8,9 \mathrm{~N}$, and $15 \mathrm{~B})$, thus falling in the group of SAD patients $(18 / 94,19 \%)$. Fold increase over post-vaccination IgG values for serotypes $8,9 \mathrm{~N}$, and $15 \mathrm{~B}$ are shown in supplemental figure S1. Of note, $16 / 18$ of the patients who were included as SAD due to a fold increase below 2 and prevaccination titers $<4 \mu \mathrm{g} / \mathrm{ml}$ had indeed pre- and postvaccination titers $>1.3 \mu \mathrm{g} / \mathrm{ml}$.
We then applied serotype-specific fifth percentile (p5) cutoff values for post-vaccination $\mathrm{IgG}$ and fold increase to the bead-based assay results, as previously determined [14]. Defining a normal response as postvaccination $\operatorname{IgG}$ value and fold increase $\geq \mathrm{p} 5$ for at least $70 \%$ of the included serotypes $(50 \%$ for children aged 2-5 years), 22 patients $(23 \%)$ could be classified as having SAD. Serotype-specific post-vaccination IgG and fold increase determined by WHO ELISA and bead-based assay are shown in Fig. 1.

When we applied the previously determined p5 cutoffs to the WHO ELISA results, two other patients scored as SAD, but 14 others were instead not identified (SAD proportion $6 \%$ ). Of these, 9 belonged to the group of SAD patients detected by bead-based assay (supplemental figure S2).

\section{S. typhi Antibody Response}

Based on our previously determined p5 values [14], we considered a normal response as post-vaccination $\mathrm{IgG}$ value $\geq$ 11.2 $\mathrm{U} / \mathrm{ml}$ and fold increase $\geq 2$ (unless pre-vaccination titer $\geq 100$ ). These criteria are comparable with those determined by other studies $[11,12,19,20]$. Five patients had prevaccination titers $\geq 11.2 \mathrm{U} / \mathrm{ml}$, but none had values $\geq 100 \mathrm{U} /$ $\mathrm{ml}$ (range 15.8-65 U/ml). Applying the aforementioned criteria, 7 patients (7\%) did not respond to the S. typhi Vi vaccination. Paired pre- and post-vaccination IgG levels and grouped post-vaccination $\mathrm{IgG}$ levels and fold increase are shown in supplemental figures S3 and S4.

\section{Allohemagglutinins}

Blood group and AHA were determined in 91 patients. Two subjects had blood group AB and no AHA. Forty-one patients had blood group A, 5 blood group B, and 43 blood group $\mathrm{O}$. Considering IgG, 10 patients had abnormal AHA titers (defined as anti-B below $1 / 2$ or anti-A below $1 / 4$, as previously published [14]). Among patients with blood group A, 3 had no anti-B AHA and 4 had low anti-B titer. One patient with blood group B had low anti-A titer, and two patients with blood group $\mathrm{O}$ had either low anti-A or anti-B titer.

\section{Comparison of Polysaccharide Antibody Responses: WHO ELISA vs. Bead-Based Assay}

We considered the 93 patients for whom both WHO ELISA and bead-based assay PPV antibody responses were available. Of 18 patients with SAD based on WHO ELISA, 10 also fall in the SAD category according to the bead-based assay results (Fisher's exact test $p=0.001$, supplemental figure S5). We then used linear regression to determine if paired results for the two serotypes analyzed by both methods ( 8 and $9 \mathrm{~N}$ ) correlated, but significant associations were found neither for $\operatorname{IgG}$ 


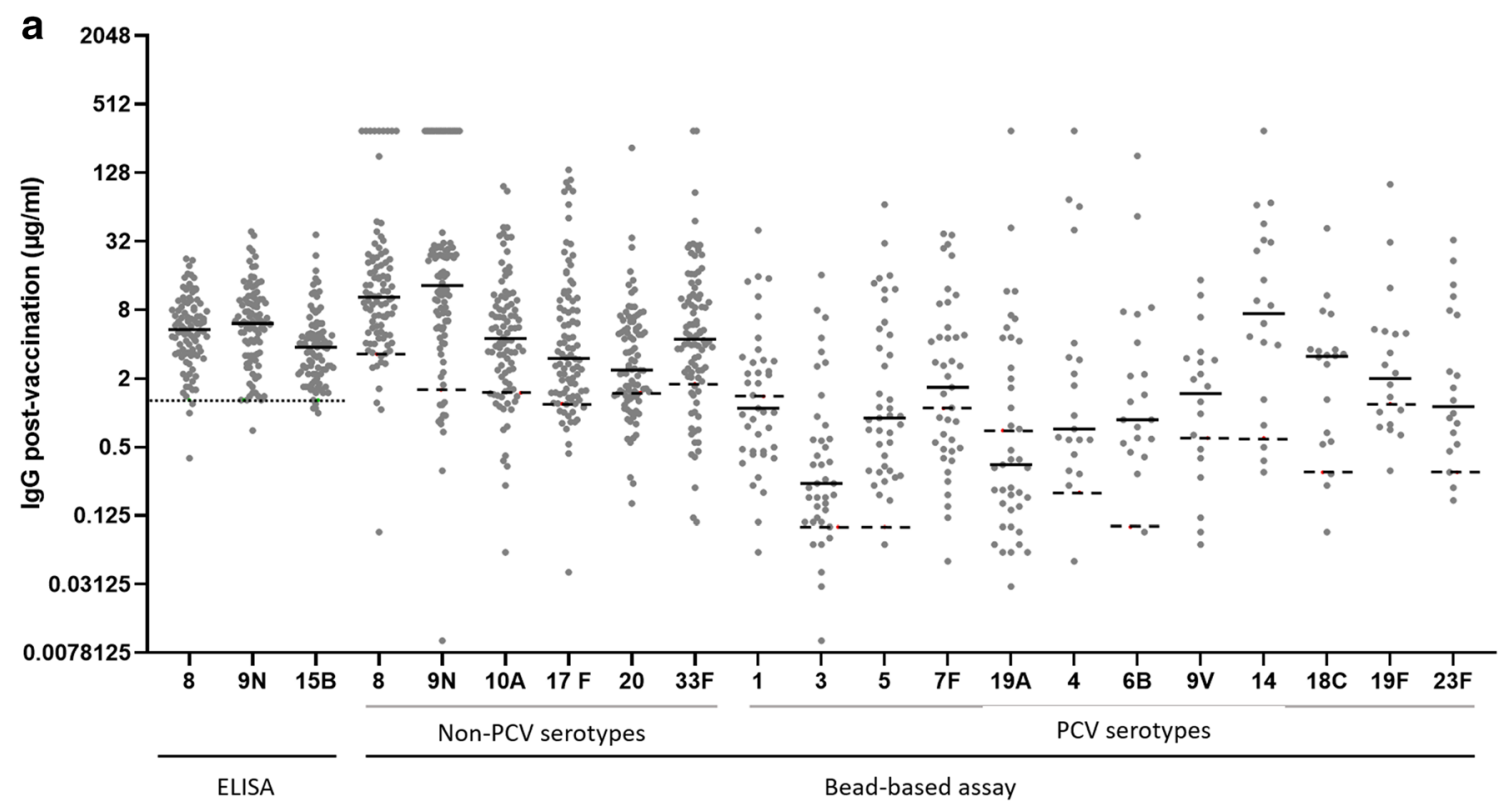

b

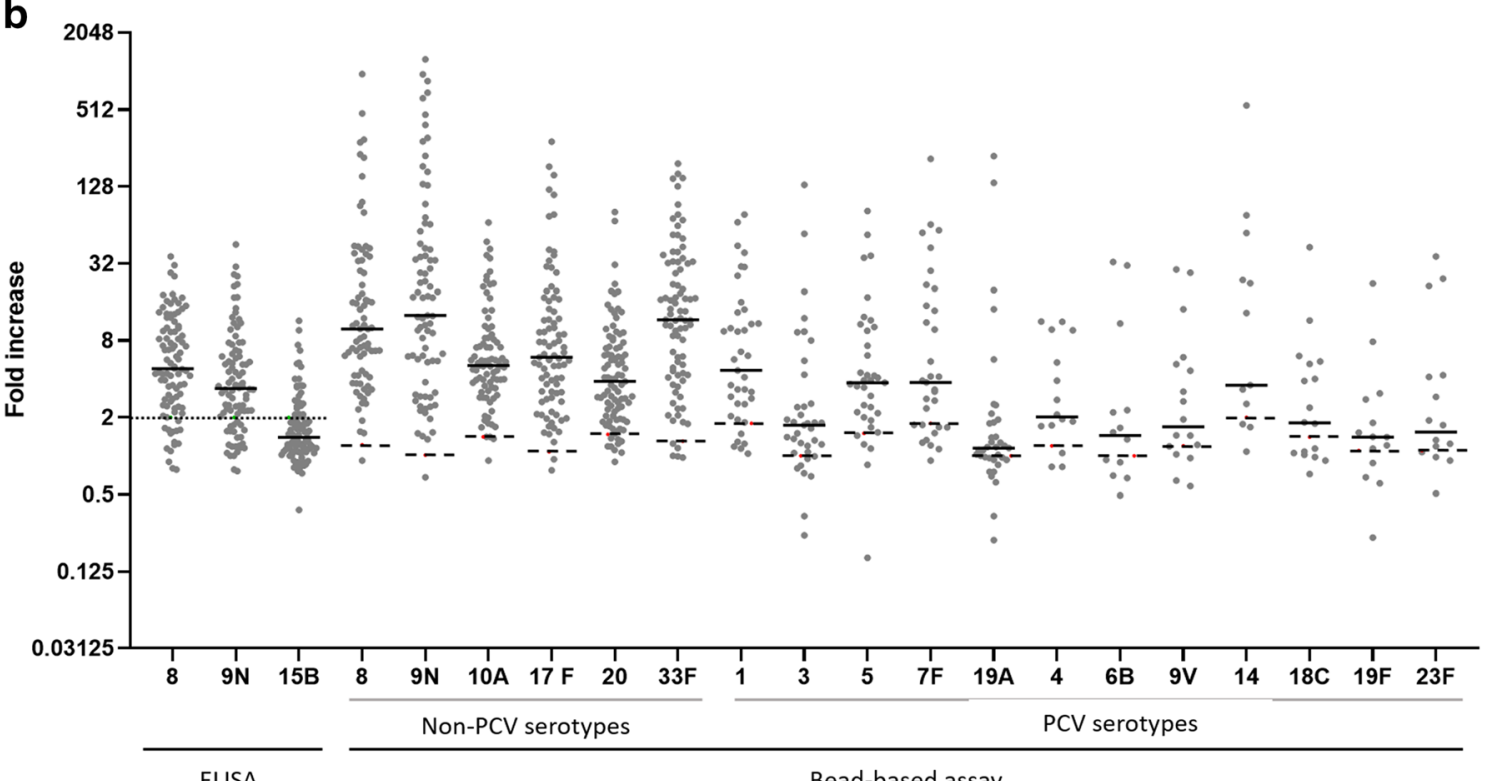

Fig. 1 Serotype-specific IgG levels (a) and fold increase (b) postvaccination with PPV, measured by WHO ELISA or bead-based assay as indicated. Dots represent single patients, solid lines indicate medians,

values $\left(r^{2} 0.12\right.$ and 0.37 for serotype 8 and $9 \mathrm{~N}$ respectively) nor fold increase post-vaccination $\left(r^{2} 0.17\right.$ and 0.13$)$. Paired results are shown in supplemental figure $\mathrm{S6}$.

\section{Comparison of Polysaccharide Antibody Responses: Can the S. typhi Antibody Response Be Used to Diagnose SAD?}

We assessed whether low pneumococcal polysaccharide response (according to WHO ELISA or bead assay) corresponded to low S. typhi polysaccharide response in our the dotted line indicates the AAAAI cutoff values for the ELISA, and dashed lines the p5 cutoff values for the bead-based assay (previously calculated as in a cohort of healthy controls [14])

cohort of patients. All subjects with SAD according to the ELISA assay or the bead-based assay and the nonresponders to $S$. typhi Vi vaccination are represented in Fig. 2 and their clinical characteristics are summarized in supplemental table S1.

Four patients had overlapping abnormal results according to all three tests. P17 is 44 years old and suffers from recurrent pneumonia and upper respiratory tract infections. P52 is 43 years old and suffers from recurrent upper respiratory tract infections and otitis. P76 is 7 years old and suffers from recurrent upper respiratory tract infections. P60 is a 2-year-old 
Fig. 2 Venn diagram showing the patients (black dots) with abnormal antibody response according to the WHO ELISA, the bead-based assay and the S. typhi antibody response. Numbers in the circles indicate number of patients. Total number of patients tested, 93

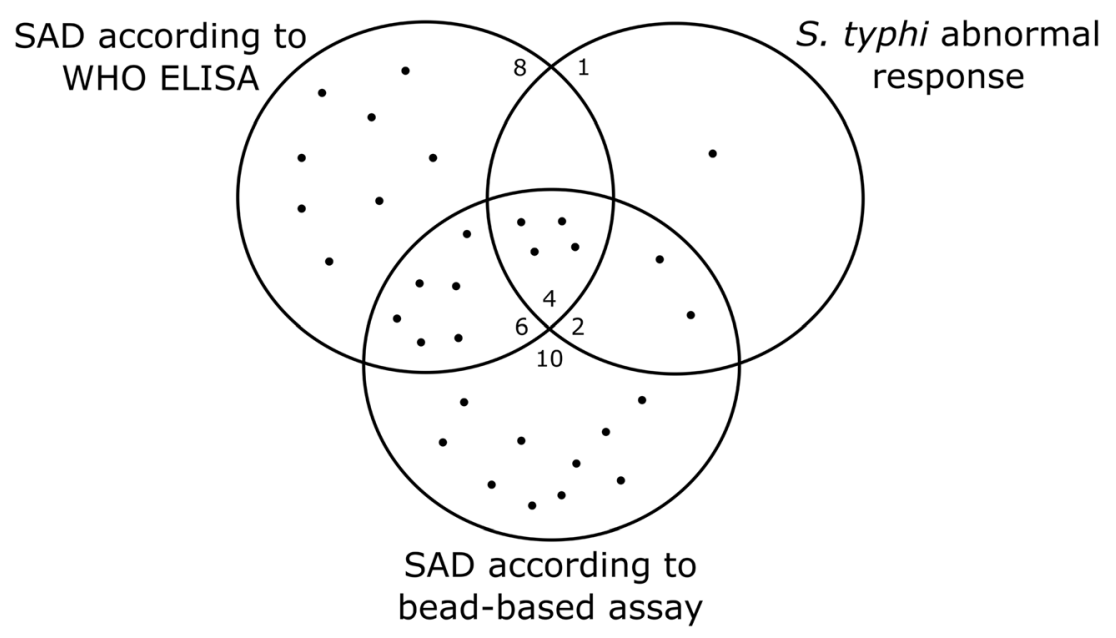

boy (later shown to carry a hypomorphic $I K B K B$ mutation, not yet published) who manifested $S$. pneumoniae sepsis and septic arthritis, $S$. pyogenes lymph node abscess and suffers from recurrent upper respiratory tract infections and otitis. He is the only subject with deficient polysaccharide response to both PPV and S. typhi Vi to display also an abnormal AHA production.

Of the remaining 3 subjects with abnormal S. typhi polysaccharide response, 2 also have SAD according to the beadbased assay. Conversely, 6 patients have SAD according to both WHO ELISA and bead-based assay but normal S. typhi polysaccharide response.

We wanted to address the question if the determination of S. typhi antibody response could assist in the diagnosis of patients affected with SAD. In our cohort, abnormal S. typhi polysaccharide response was found in 7 subjects, of whom 4 also had SAD according to WHO ELISA (4/18) and 6 had SAD according to the bead-based assay (6/22). Association of abnormal $S$. typhi antibody response and SAD was significant ( $p<0.001$ for the bead-based assay and $p=0.02$ for the WHO ELISA). We calculated the positive (ppv) and negative (npv) predictive value of $S$. typhi antibody response using the WHO ELISA or the bead-based assay as our reference test alternately. We found ppv 0.57-npv 0.84 for WHO ELISA and ppv 0.86-npv 0.82 for bead-based assay. This implies that while an abnormal $S$. typhi antibody response is mostly associated with SAD (specificity $96 \%$ based on WHO ELISA and $98 \%$ on bead-based assay), the test is not sensitive enough to identify most of the affected patients when relying on the previously established assays (sensitivity $22 \%$ based on WHO ELISA and $27 \%$ on bead-based assay, supplemental figure S5).

\section{Correlation Between AHA and Abnormal Polysaccharide Response}

When AHA results are compared with PPV and S. typhi Vi antibody responses, only the patient with a defect of IKK- $\beta$ results deficient in the production of all tested antibodies. AHA below normal ranges are present in 5 patients with SAD: $3 / 10$ subjects with SAD according to both WHO ELISA and bead-based assay and 2/8 remaining subjects with SAD according to WHO ELISA (Fig. 3). The test reveals a sensitivity of $27 \%$ or $13 \%$ and a specificity of $93 \%$ or $89 \%$ for the identification of SAD patients according to WHO ELISA or bead-based assay, respectively. The association of AHA results with $\mathrm{SAD}$ is not significant according to the bead assay $(p=0.7)$ and significant according to WHO ELISA $(p=0.02)$.

\section{Correlation Between Clinical Phenotype, Age and Abnormal Polysaccharide Response}

No correlation with age nor with the clinical presentation (considering the frequency of total infections or the number of pneumonia/otitis singularly) could be found for neither PPV nor $S$. typhi Vi antibody response.

The group of patients diagnosed with SAD included a child with recurrent invasive pyogenic infections due to IKK- $\beta$ deficiency and a child with recurrent pneumonia and early-onset bronchiectasis, in line with the textbook SAD phenotype. However, three adults manifested symptoms beyond those typically associated with SAD: two patients suffered from $\mathrm{CMC}$ in addition to pneumonia and upper respiratory tract infection, and one patient was diagnosed with granulomatous hepatitis in the context of CVID.

\section{Discussion}

The lack of consensus and of evidence regarding the diagnostic criteria of SAD represents a challenge in daily clinical practice [1]. The widely adopted AAAAI guidelines were originally based on personal communications and small patient samples, rather than large controlled data sets, thus contributing to the controversy on the definition and correct 

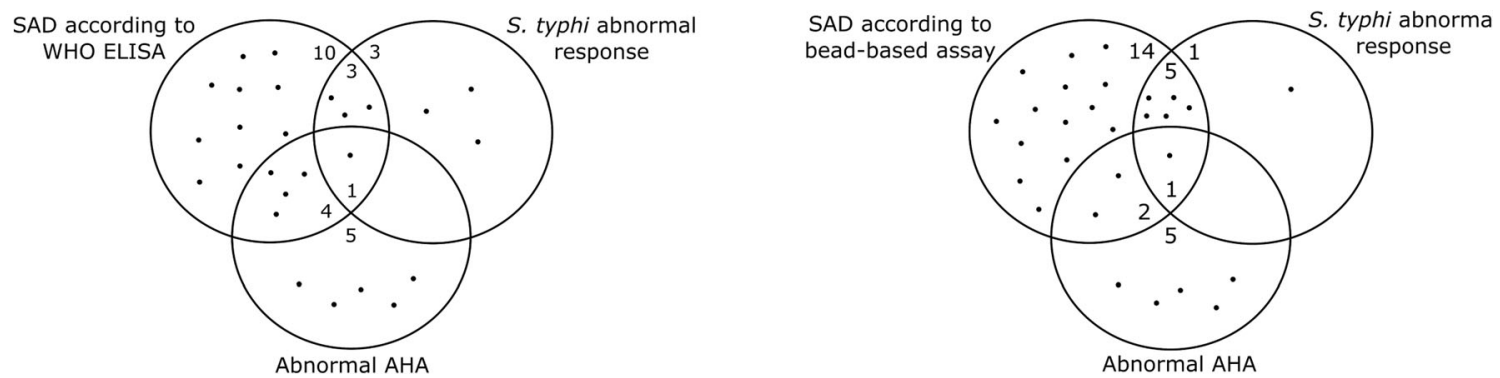

Fig. 3 Venn diagram showing the patients (black dots) with abnormal antibody response according to AHA determination, S. typhi antibody response and either the WHO ELISA or the bead-based assay, as indicated. Numbers in the circles indicate number of patients. Total number of patients tested 91

assessment of SAD [1, 10]. An additional problem is represented by the fact that many health care providers do not have access to the WHO ELISA to measure PPV responses and use bead-based assays instead. We here compared these two methods as well as the antibody responses to $S$. typhi and AHA in a large cohort of patients, including a considerable share of pediatric patients immunized with $S$. typhi Vi vaccine.

We confirm that different methods of determination of PPV antibodies do not correlate well [1, 2, 7, 8], and even if the association between SAD diagnosed by WHO ELISA and by bead-based assay is significant, several patients would be considered not-SAD when using a single method. This observation could be linked to the fact that the immunogenicity of different PPV serotypes is extremely variable, and most subjects do not respond equally to the various serotypes (Fig. 1, supplemental table S1) [14, 21]. Moreover, different capsular polysaccharides have age-dependent immunogenicity [22]. Given the younger median age of this diseased population versus the healthy population previously reported (median age 4 years and range 2-52 years in the patients vs median age 25 years and range 1055 years in the controls) [14], we would have expected an increased proportion of patients diagnosed with SAD. As this is not the case, over-interpretation as nonresponders due to younger age is unlikely [22]. To put the patients' results in perspective, $11 \%$ of the subjects from the healthy cohort could be classified as SAD according to the AAAAI criteria on WHO ELISA results, which reduced to $2 \%$ when fifth percentile cutoffs were applied, while for the patients the proportions were $19 \%$ and $6 \%$, respectively. Similarly, the bead-based assay detected $4 \%$ of SAD in this healthy population and $23 \%$ in the cohort of patients [14].

In our study, we performed the WHO ELISA for only three PPV serotypes, thus limiting the precision of our detection of non-responding individuals, and possibly explaining the partial discordance between WHO ELISA and bead-based assay results. This great interindividual variability complicates the search for a reliable method for testing PPV antibody response that ideally requires the analysis of antibodies to a large number of serotypes. Further complicating matters, the criteria proposed by AAAAI to define non-responders, such as a less than two-fold increase of the titers before/after vaccination unless the pre-vaccination titers are $>4 \mu \mathrm{g} / \mathrm{ml}$, are entirely arbitrary [6]. Among our patients, 16 out of $18 \mathrm{SAD}$ who were identified on WHO ELISA by the "less than two-fold increase" criterion actually have pre- and post-immunization titers > $1.3 \mu \mathrm{g} / \mathrm{ml}$, leaving open the possibility of a different interpretation of their antibody response. An additional hindrance is represented by the now widespread offer of pneumococcal conjugated vaccines to young children in most European and North-American countries, which elicit a combined immunological response and can thus mask a polysaccharide antibody deficiency, limiting the number of serotypes available for diagnostic PPV challenge.

For this reason, we looked into the possibility that the polysaccharide response to $S$. typhi Vi or the AHA titer could aid in the identification of SAD patients, as previously indicated in other studies $[6,11,12]$. We found that while abnormal IgG production postvaccination with $S$. typhi Vi correlates well with both WHO ELISA and bead-based assay, and while the assay has a good specificity for diagnosing SAD, it is not sensitive enough to be reliably used as a single test for the identification of patients. One of the explanations of this diversity in response may also lie in the different nature of the capsular polysaccharides of $S$. typhi and of $S$. pneumoniae. The alleged association between low AHA and SAD, still cited in textbooks and clinical guidelines despite only anecdotal evidence [6, $10]$, is currently not considered to be significant, as previously shown $[13,23]$ and confirmed in this study.

We suggest that SAD should be considered an immunological phenotype associated with a broad clinical expression, and not a well-defined end diagnosis. In our experience, the clinical manifestations of patients with SAD are varied and not restricted to the respiratory tract. In our cohort of patients with suggestive symptoms, the 
rate of diagnosis of SAD is $19-23 \%$ depending on the test, in line with most published reports [3, 24-26]. Interestingly, only one patient manifested bronchiectasis, a trait that was previously found to strongly correlate with SAD $[13,26,27]$.

To conclude, we suggest caution in the interpretation of PPV responses, until a reliable method, which may consist of assessing the function of antibodies and not only their levels, can be devised, or until more studies strengthen the evidence in support of a single assay or a combination of existing assays, e.g., the bead-based assay with $\mathrm{p} 5$ cutoffs and the $S$. typhi antibody response. Moreover, as molecular diagnoses gain further importance with time, it is probable that assays such as the measurement of polysaccharide antibody responses will become redundant or less relevant for clinical care. In line with other recommendations $[1,2]$, patients' care should rely on the correlation of the results with the clinical phenotype and evolution to determine the best therapeutic strategy, on a case-by-case basis. From a research point of view, in-depth molecular analyses of the B cells and immune system of these patients could be useful to finally unveil the origin of specific polysaccharide antibody deficiency, especially by comparing subjects with deficient responses to all or only a specific group of microbial polysaccharides.

Acknowledgments We thank Marco Baggio for his assistance with the statistical analyses.

Author Contributions HS and IM designed the study. HS conducted the experiments and revised the manuscript. GB analyzed and interpreted the data and prepared the manuscript. LM helped with data interpretation and critically revised the manuscript. IM, HS, RS, MP, KDB, BB, MB, FV, and NL enrolled the patients and critically revised the manuscript. DD, $\mathrm{BK}, \mathrm{CJ}, \mathrm{M}-\mathrm{PE}$, and $\mathrm{XB}$ performed laboratory measures and critically revised the manuscript.

Funding Information GB is supported by the Research Foundation Flanders (project G0C $8517 \mathrm{~N}$ ). HS is supported by a PhD fellowship grant of the Research Foundation - Flanders. RS is supported by a senior clinical investigator fund of the Research Foundation - Flanders. IM is supported by the Jeffrey Modell Foundation, by the Research Foundation Flanders (project G0C8517N), and is holder of the CSL Behring Chair in Primary Immunodeficiency in Children. This work is supported by a GOA grant of the KU Leuven. The Binding Site (Birmingham, UK) provided the ELISA kits for measuring $S$. typhi antibodies.

Compliance with Ethical Standards The study was approved by the ethical committee of the hospital and written informed consent was obtained from the patients or their parents.

Conflict of Interest IM is supported by a CSL Behring Chair in Primary Immunodeficiency, paid to institution. The other authors have no conflict of interest to declare.

\section{References}

1. Perez E, Bonilla FA, Orange JS, Ballow M. Specific antibody deficiency: controversies in diagnosis and management. Front Immunol. 2017;8:586.

2. Sorensen RU, Edgar D. Specific antibody deficiencies in clinical practice. J Allergy Clin Immunol Pract. 2019;7(3):801-8.

3. Boyle RJ, Le C, Balloch A, Tang ML-K. The clinical syndrome of specific antibody deficiency in children. Clin Exp Immunol. 2006;146(3):486-92.

4. ESID Registry Working Party. ESID Registry - working definitions for clinical diagnosis of PID. 22 Jan 2019. Available from: https:// esid.org/content/download/16792/456144/file/ESIDRegistry_ ClinicalCriteria.pdf .

5. Quataert SA, Kirch CS, Wiedl LJ, Phipps DC, Strohmeyer S, Cimino CO, et al. Assignment of weight-based antibody units to a human antipneumococcal standard reference serum, lot 89-S. Clin Diagn Lab Immunol. 1995;2(5):590-7.

6. Orange JS, Ballow M, Stiehm ER, Ballas ZK, Chinen J, De La Morena $\mathrm{M}$, et al. Use and interpretation of diagnostic vaccination in primary immunodeficiency: a working group report of the basic and clinical immunology interest section of the American Academy of Allergy, Asthma \& Immunology. J Allergy Clin Immunol. 2012;130(3, Supplement):S1-24.

7. Daly TM, Hill HR. Use and clinical interpretation of pneumococcal antibody measurements in the evaluation of humoral immune function. Clin Vaccine Immunol. 2015;22(2):148-52.

8. Balloch A, Licciardi PV, Tang MLK. Serotype-specific anti-pneumococcal IgG and immune competence: critical differences in interpretation criteria when different methods are used. J Clin Immunol. 2013;33(2):335-41.

9. Hajjar J, Al-Kaabi A, Kutac C, Dunn J, Shearer WT, Orange JS. Questioning the accuracy of currently available pneumococcal antibody testing. J Allergy Clin Immunol. 2018;142(4):1358-60.

10. Paris K, Sorensen RU. Assessment and clinical interpretation of polysaccharide antibody responses. Ann Allergy Asthma Immunol. 2007;99(5):462-4.

11. Sánchez-Ramón S, de Gracia J, García-Alonso AM, Rodríguez Molina JJ, Melero J, de Andrés A, et al. Multicenter study for the evaluation of the antibody response against Salmonella typhi Vi vaccination (EMPATHY) for the diagnosis of anti-polysaccharide antibody production deficiency in patients with primary immunodeficiency. Clin Immunol. 2016;169:80-4.

12. Evans C, Bateman E, Steven R, Ponsford M, Cullinane A, Shenton $\mathrm{C}$, et al. Measurement of Typhi Vi antibodies can be used to assess adaptive immunity in patients with immunodeficiency. Clin Exp Immunol. 2018;192(3):292-301.

13. Schaballie H, Vermeulen F, Verbinnen B, Frans G, Vermeulen E, Proesmans $\mathrm{M}$, et al. Value of allohaemagglutinins in the diagnosis of a polysaccharide antibody deficiency. Clin Exp Immunol. 2015;180(2):271-9.

14. Schaballie H, Bosch B, Schrijvers R, Proesmans M, De Boeck K, Boon $\mathrm{MN}$, et al. Fifth percentile cutoff values for antipneumococcal polysaccharide and anti-Salmonella typhi vi IgG describe a normal polysaccharide response. Front Immunol. 2017;8:546.

15. Jeurissen A, Moens L, Raes M, Wuyts G, Willebrords L, Sauer K, et al. Laboratory diagnosis of specific antibody deficiency to pneumococcal capsular polysaccharide antigens. Clin Chem. 2007;53(3):505-10.

16. Schaballie H, Wuyts G, Dillaerts D, Frans G, Moens L, Proesmans $\mathrm{M}$, et al. Effect of previous vaccination with pneumococcal conjugate vaccine on pneumococcal polysaccharide vaccine antibody responses. Clin Exp Immunol. 2016;185(2):180-9. 
17. Lal G, Balmer P, Stanford E, Martin S, Warrington R, Borrow R. Development and validation of a nonaplex assay for the simultaneous quantitation of antibodies to nine Streptococcus pneumoniae serotypes. J Immunol Methods. 2005;296(1):135-47.

18. Concepcion N, Frasch CE. Evaluation of previously assigned antibody concentrations in pneumococcal polysaccharide reference serum $89 \mathrm{SF}$ by the method of cross-standardization. Clin Diagn Lab Immunol. 1998;5(2):199-204.

19. Ferry BL, Misbah SA, Stephens P, Sherrell Z, Lythgoe H, Bateman E, et al. Development of an anti-Salmonella typhi Vi ELISA: assessment of immunocompetence in healthy donors. Clin Exp Immunol. 2004;136(2):297-303.

20. Bausch-Jurken MT, Verbsky JW, Gonzaga KA, Elms NP, Hintermeyer MK, Gauld SB, et al. The use of Salmonella typhim vaccine to diagnose antibody deficiency. J Clin Immunol. 2017;37(5):427-33.

21. Uddin S, Borrow R, Haeney MR, Moran A, Warrington R, Balmer $\mathrm{P}$, et al. Total and serotype-specific pneumococcal antibody titres in children with normal and abnormal humoral immunity. Vaccine. 2006;24(27):5637-44.

22. Bossuyt X, Borgers H, Moens L, Verbinnen B, Meyts I. Age- and serotype-dependent antibody response to pneumococcal polysaccharides. J Allergy Clin Immunol. 2011;127(4):1079-80.
23. Cheng YK, Decker PA, O'Byrne MM, Weiler CR. Clinical and laboratory characteristics of 75 patients with specific polysaccharide antibody deficiency syndrome. Ann Allergy Asthma Immunol. 2006;97(3):306-11.

24. Javier FC, Moore CM, Sorensen RU. Distribution of primary immunodeficiency diseases diagnosed in a pediatric tertiary hospital. Ann Allergy Asthma Immunol. 2000;84(1):25-30.

25. Sanders LAM, Rijkers GT, Kuis W, Tenbergen-Meekes AJ, de Graeff-Meeder BR, Hiemstra I, et al. Defective antipneumococcal polysaccharide antibody response in children with recurrent respiratory tract infections. J Allergy Clin Immunol. 1993;91(1, Part 1): $110-9$.

26. Schatorjé EJH, de Jong E, van Hout RWNM, García Vivas Y, de Vries E. The challenge of immunoglobulin-G subclass deficiency and specific polysaccharide antibody deficiency - a Dutch pediatric cohort study. J Clin Immunol. 2016;36(2):141-8.

27. Slade CA, Bosco JJ, Binh Giang T, Kruse E, Stirling RG, Cameron $\mathrm{PU}$, et al. Delayed diagnosis and complications of predominantly antibody deficiencies in a cohort of Australian adults. Front Immunol. 2018;9:694.

Publisher's Note Springer Nature remains neutral with regard to jurisdictional claims in published maps and institutional affiliations. 